\title{
CHOW GROUPS OF INTERSECTIONS OF QUADRICS VIA HOMOLOGICAL PROJECTIVE DUALITY AND (JACOBIANS OF) NONCOMMUTATIVE MOTIVES
}

\author{
MARCELLO BERNARDARA AND GONÇALO TABUADA
}

\begin{abstract}
The Beilinson-Bloch type conjectures predict that the low degree rational Chow groups of intersections of quadrics are one dimensional. This conjecture was proved by Otwinowska in [27]. Making use of homological projective duality and the recent theory of (Jacobians of) noncommutative motives, we give an alternative proof of this conjecture in the case of a complete intersection of either two quadrics or three odd-dimensional quadrics. Moreover, without the use of the powerful Lefschetz theorem, we prove that in these cases the unique non-trivial algebraic Jacobian is the middle one. As an application, making use of Vial's work [33, 34], we describe the rational Chow motives of these complete intersections and show that smooth fibrations in such complete intersections over small dimensional bases $S$ verify Murre's conjecture $(\operatorname{dim}(S) \leq 1)$, Grothendieck's standard conjectures $(\operatorname{dim}(S) \leq 2)$, and Hodge's conjecture $(\operatorname{dim}(S) \leq 3)$.
\end{abstract}

\section{Statement of Results}

Let $k$ be a field and $X$ a smooth projective $k$-subscheme of $\mathbb{P}^{n}$. Whenever $X$ is a complete intersection of multidegree $\left(d_{1}, \ldots, d_{r}\right)$ one has the numerical invariant

$$
\kappa:=\left[\frac{n-\sum_{j=2}^{r} d_{j}}{d_{1}}\right],
$$

where [-] denotes the integral part of a rational number. A careful analysis of the different Weil cohomology theories of $X$ led to the following conjecture of BeilinsonBloch type (explicitly stated by Paranjape in [28, Conjecture 1.8]):

Conjecture 1.1. There is an isomorphism $C H_{i}(X)_{\mathbb{Q}} \simeq \mathbb{Q}$ for every $i<\kappa$.

Otwinowska proved Conjecture 1.1 in the case where $X$ is a complete intersection of quadrics, i.e., when $d_{1}=\cdots=d_{r}=2$; see [27, Cor. 1]. If one further assumes that $\kappa=[\operatorname{dim}(X) / 2]$, and that $k \subseteq \mathbb{C}$ is algebraically closed, then Conjecture 1.1 admits an alternative proof. Concretely, our first main result is the following:

Theorem 1.2. Conjecture 1.1 holds when:

(i) $X$ is a complete intersection of two quadrics;

(ii) $X$ is a complete intersection of three odd-dimensional quadrics.

Otwinowska's proof is based on a geometric recursive argument. First, one establishes the induction step: if Conjecture 1.1 holds for complete intersections of multidegree $\left(d_{1}, \ldots, d_{r}\right)$, then it also holds for complete intersections of multidegree

Date: May 4, 2015.

2000 Mathematics Subject Classification. 14A22, 14C15, 14F05, 14J40, 14M10.

G. Tabuada was partially supported by a NSF CAREER Award. 
$\left(d_{1}, \ldots, d_{r}, d_{r}\right)$; see [27, Theorem 1]. Then, one uses the fact that Conjecture 1.1 is known in the case of quadric hypersurfaces. One should also mention the work of Esnault-Levine-Viehweg [11]. In loc. cit., a geometric proof of Conjecture 1.1 for very small values of $i$ was obtained via a generalization of Roitman's techniques.

Our proof of Theorem 1.2 is intrinsically different. It is "categorical" in nature and based on recent technology such as Kuznetsov's homological projective duality and (Jacobians of) noncommutative motives. In contrast with the proof of Otwinowska, and of Esnault-Levine-Viehweg, it highlights the geometric information contained in the derived category of $X$ and on its noncommutative motive.

Now, recall from Griffiths [13] the construction of the Jacobians $J^{i}(X), 0 \leq i \leq$ $\operatorname{dim}(X)-1$. In contrast with the Picard $J^{0}(X)=\operatorname{Pic}^{0}(X)$ and the Albanese $J^{\operatorname{dim}(X)-1}(X)=\operatorname{Alb}(X)$ varieties, the intermediate Jacobians are not (necessarily) algebraic. Nevertheless, they contain an algebraic torus $J_{a}^{i}(X) \subseteq J^{i}(X)$ defined by the image of the Abel-Jacobi map

$$
A J^{i}: A^{i+1}(X)_{\mathbb{Z}} \rightarrow J^{i}(X) \quad 0 \leq i \leq \operatorname{dim}(X)-1,
$$

where $A^{i+1}(X)_{\mathbb{Z}}$ stands for the group of algebraic trivial cycles of codimension $i+1$; consult Voisin $[35, \S 12]$ for further details. In the odd-dimensional cases, i.e., when $\operatorname{dim}(X)=2 \kappa+1$, the following holds:

(i) When $X$ is a complete intersection of two even-dimensional quadrics, Reid proved in [30, Theorem 4.14] that $J_{a}^{\kappa}(X)$ is isomorphic, as a principally polarized abelian variety, to the Jacobian $J(C)$ of the hyperelliptic curve $C$ naturally associated to $X$;

(ii) When $X$ is a complete intersection of three odd-dimensional quadrics, Beauville proved in proved in [3, Theorem 6.3] that $J_{a}^{\kappa}(X)$ is isomorphic, as a principally polarized abelian variety, to the $\operatorname{Prym}$ variety $\operatorname{Prym}(\widetilde{C} / C)$ naturally associated to $X$.

Let $X$ be a complete intersection of either two quadrics of three odd-dimensional quadrics. Making use of Veronese embedding, we can apply Lefschetz theorem (see $[35,13.2 .3])$ to conclude ${ }^{1}$ that $H^{p, q}(X)=0$ if $p \neq q$ and $p+q \neq \kappa$. This implies that the above Jacobians $J_{a}^{\kappa}(X)$ are the only non-trivial ones. Our second main result is a proof of this latter fact which avoids the use of the powerful Lefschetz theorem and Paranjape's results:

Theorem 1.4. (i) When $X$ is a complete intersection of two odd-dimensional quadrics, we have $J_{a}^{i}(X)=0$ for every $i$;

(ii) When $X$ is a complete intersection of either two even-dimensional quadrics of three odd-dimensional quadrics, we have $J_{a}^{i}(X)=0$ for every $i \neq \kappa$.

\section{Applications}

Our first application, obtained by combining Theorems 1.2 and 1.4 with Vial's work [33] on intermediate algebraic Jacobians, is the following:

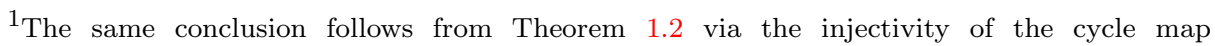
$C H_{i}(X)_{\mathbb{Q}} \rightarrow H^{2 \kappa+1-i}(X, \mathbb{Q})$ and Paranjape's results [28, Prop. 6.4] (see also [27, Thm. 1]).
} 
Corollary 2.1. Let $X$ be a complete intersection of two odd-dimensional quadrics. In this case we have the following motivic decomposition

$$
M^{i}(X)_{\mathbb{Q}} \simeq \begin{cases}\mathbf{L}^{\otimes \frac{i}{2}} & \text { if } 0 \leq i \leq 2 d, i \neq d, \text { i even } \\ \left(\mathbf{L}^{\otimes \frac{d}{2}}\right)^{\oplus(d+4)} & \text { if } i=d \\ 0 & \text { otherwise, }\end{cases}
$$

where $d:=\operatorname{dim}(X)$ and $\mathbf{L}$ is the rational Lefschetz motive.

Let $X$ be a complete intersection of either two even-dimensional quadrics or three odd-dimensional quadrics. In these cases we have the motivic decomposition

$$
M^{i}(X)_{\mathbb{Q}} \simeq \begin{cases}\mathbf{L}^{\otimes \frac{i}{2}} & \text { if } 0 \leq i \leq 2 d, i \text { even } \\ M^{1}\left(J_{a}^{\kappa}(X)\right)_{\mathbb{Q}}(\kappa-d) & \text { if } i=d \\ 0 & \text { otherwise. }\end{cases}
$$

Moreover, in all the above cases the rational Chow motive $M(X)_{\mathbb{Q}}$ is Kimura-finite.

Intuitively speaking, Corollary 2.1 is the "motivic refinement" of Theorems 1.2 and 1.4. Our second application, obtained by combining Theorem 1.2 with Vial's work [34] on fibrations, is the following:

Corollary 2.4. Let $f: Y \rightarrow B$ be a smooth dominant flat morphism between smooth projective $k$-schemes. Assume that the fibers of $f$ are either complete intersections of two quadrics or complete intersections of three odd-dimensional quadrics. Under these assumptions, the following holds:

(i) When $\operatorname{dim}(B) \leq 1$, the rational Chow motive $M(Y)_{\mathbb{Q}}$ is Kimura-finite and $Y$ satisfies Murre's conjectures;

(ii) When $\operatorname{dim}(B) \leq 2, Y$ satisfies Grothendieck's standard conjectures;

(iii) When $\operatorname{dim}(B) \leq 3, Y$ satisfies Hodge's conjecture.

Notations: Throughout the article we will work over an algebraically closed field $k \subset \mathbb{C}$. All $k$-schemes will be smooth and projective. Given a $k$-scheme $X$, we will write $C H_{i}(X)_{\mathbb{Z}}$ for the Chow group of $i$-dimensional cycles modulo rational equivalence and $A_{i}(X)_{\mathbb{Z}}$ for the subgroup of algebraically trivial cycles. We will also use the codimensional notations $C H^{i}(X)_{\mathbb{Z}}$ and $A^{i}(X)_{\mathbb{Z}}$ and rational coefficients $C H_{i}(X)_{\mathbb{Q}}, A_{i}(X)_{\mathbb{Q}}, C H^{i}(X)_{\mathbb{Q}}, A^{i}(X)_{\mathbb{Q}}$. Finally, the derived category of perfect complexes of $X$ will be denoted by $\operatorname{perf}(X)$. Note that the canonical inclusion of categories $\operatorname{perf}(X) \hookrightarrow \mathcal{D}^{b}(\operatorname{Coh}(X))$ is an equivalence since $X$ is smooth.

Acknowledgments: The authors are very grateful to Hélène Esnault for precious advices, and to Charles Vial and Asher Auel for useful comments and answers.

\section{Preliminaries}

Differential graded categories. We will assume that the reader is familiar with the language of differential graded $(=\mathrm{dg})$ categories. The standard reference is Keller's ICM survey [16]. Every (dg) $k$-algebra $A$ gives naturally rise to a dg category $\underline{A}$ with a single object and $(\mathrm{dg}) k$-algebra of endomorphisms $A$. Another source of examples is provided by $k$-schemes since, as proved by Lunts-Orlov in [23, Theorem 2.12], the derived category of perfect complexes perf $(X)$ admits a unique $\mathrm{dg}$ enhancement $\operatorname{perf}^{\mathrm{dg}}(X)$. Recall from Kontsevich $[17,18,19]$ that a dg category $\mathcal{A}$ is called smooth if it is perfect as a bimodule over itself and proper if for each ordered pair of objects $(x, y)$ we have $\sum_{i} \operatorname{dim} H^{i} \mathcal{A}(x, y)<\infty$. The main examples are the dg categories $\operatorname{perf}^{\mathrm{dg}}(X)$. 
Noncommutative Chow motives. Recall from the survey article [31] the construction of the category $\operatorname{NChow}(k)_{\mathbb{Q}}$ of noncommutative Chow motives (with rational coefficients) and of the $\otimes$-functor

$$
U(-)_{\mathbb{Q}}: \operatorname{spdgcat}(k) \longrightarrow \operatorname{NChow}(k)_{\mathbb{Q}},
$$

where $\operatorname{spdgcat}(k)$ stands for the category of smooth proper dg categories. Given a $k$-scheme $X$, we will write $N C(X)_{\mathbb{Q}}$ instead of $U\left(\operatorname{perf}^{\mathrm{dg}}(X)\right)_{\mathbb{Q}}$.

Proposition 3.1. Every semi-orthogonal decomposition $\operatorname{perf}(X)=\left\langle\mathcal{T}_{1}, \ldots, \mathcal{T}_{r}\right\rangle$ gives rise to a direct sum decomposition $N C(X)_{\mathbb{Q}} \simeq U\left(\mathcal{T}_{1}^{\mathrm{dg}}\right)_{\mathbb{Q}} \oplus \cdots \oplus U\left(\mathcal{T}_{r}^{\mathrm{dg}}\right)_{\mathbb{Q}}$, where $\mathcal{T}_{i}^{\mathrm{dg}}$ stands for the $d g$ enhancement of $\mathcal{T}_{i}$ induced from $\operatorname{perf}^{\mathrm{dg}}(X)$.

Proof. By construction of the functor $U(-)_{\mathbb{Q}}$, every semi-orthogonal decomposition of $\operatorname{perf}(X)=\left\langle\mathcal{T}, \mathcal{T}^{\perp}\right\rangle$ of length 2 gives rise to a direct sum decomposition $N C(X)_{\mathbb{Q}} \simeq U\left(\mathcal{T}^{\mathrm{dg}}\right)_{\mathbb{Q}} \oplus U\left(\mathcal{T}^{\perp, \mathrm{dg}}\right)_{\mathbb{Q}} ;$ consult [32, Theorem 6.3] for details. The proof follows now from a recursive argument applied to the semi-orthogonal decompositions $\left\langle\left\langle\mathcal{T}_{i}\right\rangle,\left\langle\mathcal{T}_{i+1}, \ldots, \mathcal{T}_{r}\right\rangle\right\rangle, 1 \leq i \leq r-1$.

\section{Jacobians of noncommutative Chow motives}

Recall from André $[1, \S 4]$ the construction of the category Chow $(k)_{\mathbb{Q}}$ of Chow motives (with rational coefficients) and of the contravariant $\otimes$-functor

$$
M(-)_{\mathbb{Q}}: \operatorname{SmProj}(k)^{\text {op }} \longrightarrow \operatorname{Chow}(k)_{\mathbb{Q}},
$$

where $\operatorname{SmProj}(k)$ stands for the category of smooth projective $k$-schemes. De Rham cohomology factors through Chow motives. Hence, given an irreducible $k$-scheme $X$ of dimension $d$, one defines

$$
N H_{d R}^{2 i+1}(X):=\sum_{C, \gamma_{i}} \operatorname{Im}\left(H_{d R}^{1}(C) \stackrel{H_{d R}^{1}\left(\gamma_{i}\right)}{\longrightarrow} H_{d R}^{2 i+1}(X)\right) \quad 0 \leq i \leq d-1,
$$

where $C$ is a curve and $\gamma_{i}: M(C)_{\mathbb{Q}} \rightarrow M(X)_{\mathbb{Q}}(i)$ a morphism in Chow $(k)_{\mathbb{Q}}$. Intuitively speaking, (4.1) are the odd pieces of de Rham cohomology that are generated by curves. By restricting the classical intersection bilinear pairings on de Rham cohomology (see $[1, \S 3.3])$ to these pieces one then obtains

$$
\langle-,-\rangle: N H_{d R}^{2 d-2 i-1}(X) \times N H_{d R}^{2 i+1}(X) \rightarrow k \quad 0 \leq i \leq d-1 .
$$

Now, recall from [25, Theorem 1.3] the construction of the Jacobian functor

$$
\mathbf{J}(-): \operatorname{NChow}(k)_{\mathbb{Q}} \longrightarrow \operatorname{Ab}(k)_{\mathbb{Q}}
$$

with values in the category of abelian varieties up to isogeny. As proved in [25, Theorem 1.7], whenever the above pairings (4.2) are non-degenerate, one has an isomorphism $\mathbf{J}\left(\operatorname{perf}^{\mathrm{dg}}(X)\right) \simeq \prod_{i=0}^{d-1} J_{a}^{i}(X)$ in $\mathrm{Ab}(k)_{\mathbb{Q}}$. As explained in loc. cit., this is always the case for $i=0$ and $i=d-1$ and all the remaining cases follow from Grothendieck's standard conjecture of Lefschetz type.

Categorical data. Let $X$ and $Y$ be two irreducible $k$-schemes of dimensions $d_{X}$ and $d_{Y}$, respectively. Assume that they are related by the categorical data:

$(\star)$ There exist semi-orthogonal decompositions $\operatorname{perf}(X)=\left\langle\mathcal{T}_{X}, \mathcal{T}_{X}^{\perp}\right\rangle$ and $\operatorname{perf}(Y)=$ $\left\langle\mathcal{T}_{Y}, \mathcal{T}_{Y}^{\perp}\right\rangle$ and an equivalence $\phi: \mathcal{T}_{X} \stackrel{\sim}{\rightarrow} \mathcal{T}_{Y}$ of triangulated categories.

Let $\Phi$ be the composition $\operatorname{perf}(X) \rightarrow \mathcal{T}_{X} \stackrel{\phi}{\simeq} \mathcal{T}_{Y} \hookrightarrow \operatorname{perf}(Y)$. 
Theorem 4.3. ([6, Theorem 2.2]) Assume that the bilinear pairings (4.2) (associated to $X$ and $Y$ ) are non-degenerate and that $\Phi$ is of Fourier-Mukai type.

(i) Under the above assumptions, one obtains a well-defined morphism $\tau$ : $\prod_{i=0}^{d_{X}-1} J_{a}^{i}(X) \rightarrow \prod_{i=0}^{d_{Y}-1} J_{a}^{i}(Y)$ in $\mathrm{Ab}(k)_{\mathbb{Q}}$.

(ii) Assume moreover that $\mathbf{J}\left(U\left(\mathcal{T}_{X}^{\perp, \mathrm{dg}}\right)_{\mathbb{Q}}\right)=0$. This holds for instance whenever $\mathcal{T}_{\bar{X}}^{\perp}$ admits a full exceptional collection. Under this extra assumption, the morphism $\tau$ is split injective.

(iii) Assume furthermore that $\mathbf{J}\left(U\left(\mathcal{T}_{Y}^{\perp, \mathrm{dg}}\right)_{\mathbb{Q}}\right)=0$. Under this extra assumption, the morphism $\tau$ becomes an isomorphism.

Remark 4.4. Theorem 4.3 was proved in loc. cit. in the case where $k=\mathbb{C}$. The same proof works mutatis mutandis for every algebraically closed field $k \subseteq \mathbb{C}$.

\section{QUADRIC FIBRATIONS AND HOMOLOGICAL PROJECTIVE DUALITY}

Quadric fibrations. Let $q: Q \rightarrow S$ be a flat quadric fibration of relative dimension $n-1, \mathcal{C}_{0}$ the sheaf of even parts of the associated Clifford algebra, and $\operatorname{perf}\left(S, \mathcal{C}_{0}\right)$ the derived category of perfect complexes over $\mathcal{C}_{0}$. As proved by Kuznetsov in [21, Theorem 4.2], one has the following semi-orthogonal decomposition

$$
\operatorname{perf}(Q)=\left\langle\operatorname{perf}\left(S, \mathcal{C}_{0}\right), \operatorname{perf}(S)_{1}, \ldots, \operatorname{perf}(S)_{n-1}\right\rangle,
$$

where $\operatorname{perf}(S)_{i}:=q^{*} \operatorname{perf}(S) \otimes \mathcal{O}_{Q / S}(i)$. Note that $\operatorname{perf}(S)_{i} \simeq \operatorname{perf}(S)$ for every $i$.

In the case where the discriminant $\Delta$ of $Q$ is smooth, the category $\operatorname{perf}\left(S, \mathcal{C}_{0}\right)$ admits a more geometric description. Concretely, when $n$ is odd (resp. even), we have a double cover $\widetilde{S} \rightarrow S$ ramified along $\Delta$ (resp. a root stack $\widehat{S}$ with a $\mathbb{Z} / 2 \mathbb{Z}$-action along $\Delta$ ), $\mathcal{C}_{0}$ lifts to an Azumaya algebra with class $\alpha$ in $\operatorname{Br}(\widetilde{S})$ (resp. in $\operatorname{Br}(\widehat{S}))$, and $\operatorname{perf}\left(S, \mathcal{C}_{0}\right) \simeq \operatorname{perf}(\widetilde{S}, \alpha)\left(\operatorname{resp} . \operatorname{perf}\left(S, \mathcal{C}_{0}\right) \simeq \operatorname{perf}(\widehat{S}, \alpha)\right)$; consult Kuznetsov [21, §3] for further details.

Intersection of quadrics. Let $X$ be a smooth complete intersection of $r$ quadric hypersurfaces in $\mathbb{P}^{n}=\mathbb{P}(V)$. The linear span of these $r$ quadrics gives rise to a hypersurface $Q \subset \mathbb{P}^{r-1} \times \mathbb{P}^{n}$, and the projection into the first factor to a flat quadric fibration $q: Q \rightarrow \mathbb{P}^{r-1}$ of relative dimension $n-1$; consult $[21, \S 5]$ for details. As above, we can then consider the sheaf $\mathcal{C}_{0}$ of even parts of the associated Clifford algebra and the derived category perf $\left(\mathbb{P}^{r-1}, \mathcal{C}_{0}\right)$ of perfect complexes.

Homological projective duality relates the structure of $\operatorname{perf}(X)$ with the structure of $\operatorname{perf}\left(\mathbb{P}^{r-1}, \mathcal{C}_{0}\right)$. Concretely, when $2 r<n+1$, one has a fully-faithful functor $\operatorname{perf}\left(\mathbb{P}^{r-1}, \mathcal{C}_{0}\right) \hookrightarrow \operatorname{perf}(X)$ and the following semi-orthogonal decomposition

$$
\operatorname{perf}(X)=\left\langle\operatorname{perf}\left(\mathbb{P}^{r-1}, \mathcal{C}_{0}\right), \mathcal{O}_{X}(1), \ldots, \mathcal{O}_{X}(n-2 r+1)\right\rangle ;
$$

see $[21, \S 5]$. Here are some (low degree) examples:

Example 5.3. (Two odd-dimensional quadrics) Let $r=2$ and $n$ even, so that $q: Q \rightarrow \mathbb{P}^{1}$ has odd relative dimension $n-1$. Thanks to the work of Kuznetsov [21, Corollary 5.7], perf $\left(\mathbb{P}^{1}, \mathcal{C}_{0}\right)$ is equivalent to the category perf $\left(\widehat{\mathbb{P}^{1}}\right)$ of the root stack with a $\mathbb{Z} / 2 \mathbb{Z}$ stacky structure on the critical points of $Q \rightarrow \mathbb{P}^{1}$. Indeed, in this case $\mathcal{C}_{0}$ lifts to a Morita-trivial Azumaya algebra.

Example 5.4. (Two even-dimensional quadrics) Let $r=2$ and $n$ odd, so that $q: Q \rightarrow \mathbb{P}^{1}$ has even relative dimension $n-1$. Let us denote by $C$ the hyperelliptic 
curve naturally associated to $X$; see $[30, \S 4]$. Similarly to Example $5.3, \operatorname{perf}\left(\mathbb{P}^{1}, \mathcal{C}_{0}\right)$ is equivalent to the category perf $(C)$; see [7, Theorem 2.9] and [21, Corollary 5.7].

Example 5.5. (Three odd-dimensional quadrics) Let $r=3$ and $n$ even, so that $q: Q \rightarrow \mathbb{P}^{2}$ has odd relative dimension $n-1$. Consider the discriminant divisor of the fibration $q$, which is a curve with at most nodal singularities; see [3, Proposition 1.2]. Let us write $C$ for the normalization of this divisor. As explained in [3, Proposition 1.5], $C$ comes equipped with an étale double covering $\widetilde{C} \rightarrow C$.

\section{Proof of Theorem 1.2 - CASe (i)}

Let $X$ be a complete intersection of two quadric hypersurfaces in $\mathbb{P}^{n}$ with $n \geq 3$. The linear span of these two quadrics gives rise to a flat quadric fibration $q: Q \rightarrow \mathbb{P}^{1}$ of relative dimension $n-1$. As explained in $\S 5$, we hence obtain the following semiorthogonal decomposition

$$
\operatorname{perf}(X)=\left\langle\operatorname{perf}\left(\mathbb{P}^{1}, \mathcal{C}_{0}\right), \mathcal{O}_{X}(1), \ldots, \mathcal{O}_{X}(n-3)\right\rangle .
$$

Two odd-dimensional quadrics. Let $n=2 m$ for some integer $m \geq 2$. Since we are intersecting two odd-dimensional quadrics, we have $\operatorname{dim}(X)=2 m-2$ and $\kappa=m-1$. Hence, in order to prove Theorem 1.2, we need to show that

$$
C H_{i}(X)_{\mathbb{Q}} \simeq \mathbb{Q} \text { for } i<m-1 .
$$

As mentioned in the above Example 5.3, one has $\operatorname{perf}\left(\mathbb{P}^{1}, \mathcal{C}_{0}\right) \simeq \operatorname{perf}\left(\widehat{\mathbb{P}^{1}}\right)$ where $\widehat{\mathbb{P}^{1}}$ is the root stack associated to the quadric fibration $q: Q \rightarrow \mathbb{P}^{1}$. Moreover, as proved by Polishchuk in [29, Theorem 1.2], perf( $\left(\widehat{\mathbb{P}^{1}}\right)$ admits a full exceptional collection of length $p+2$, where $p$ stands for the number of discriminant points. Thanks to Reid [30, Proposition 2.1], we have $p=n+1=2 m+1$. Hence, using Proposition 3.1 and the above semi-orthogonal decomposition (6.1), we obtain

$$
N C(X)_{\mathbb{Q}} \simeq U\left(\operatorname{perf}\left(\mathbb{P}^{1}, \mathcal{C}_{0}\right)^{\mathrm{dg}}\right)_{\mathbb{Q}} \oplus \mathbf{1}_{\mathbb{Q}}^{\oplus 2 m-3} \simeq \mathbf{1}_{\mathbb{Q}}^{\oplus 2 m+3} \oplus \mathbf{1}_{\mathbb{Q}}^{\oplus 2 m-3} \simeq \mathbf{1}_{\mathbb{Q}}^{\oplus 4 m}
$$

where $\mathbf{1}_{\mathbb{Q}}:=U(\underline{k})$ stands for the $\otimes$-unit of $\operatorname{NChow}(k)_{\mathbb{Q}}$.

Proposition 6.4. The Chow motive $M(X)_{\mathbb{Q}}$ is of $\mathbb{Q}$-Lefschetz type and $C H^{*}(X)_{\mathbb{Q}}$ is a finite $4 m$-dimensional $\mathbb{Q}$-vector space ${ }^{2}$.

Proof. A noncommutative Chow motive is called of $\mathbb{Q}$-unit type if it is isomorphic in $\operatorname{NChow}(k)_{\mathbb{Q}}$ to $\oplus_{i=1}^{r} \mathbf{1}_{\mathbb{Q}}$ for some $r>0$. Thanks to the above isomorphisms (6.3), $N C(X)_{\mathbb{Q}}$ is of $\mathbb{Q}$-unit type with $r=4 m$. Hence, following [25, Theorems 1.3 and 1.7], the rational Chow motive $M(X)_{\mathbb{Q}}$ is of $\mathbb{Q}$-Lefschetz type. Moreover, there exists a choice of integers $l_{1}, \ldots, l_{4 m} \in\{0, \ldots, 2 m-2\}$ giving rise to an isomorphism

$$
M(X)_{\mathbb{Q}} \simeq \mathbf{L}^{\otimes l_{1}} \oplus \cdots \oplus \mathbf{L}^{\otimes l_{4 m}} .
$$

Using (6.5) we conclude automatically that $C H^{*}(X)_{\mathbb{Q}}$ is a finite $4 m$-dimensional $\mathbb{Q}$-vector space. This achieves the proof.

\footnotetext{
${ }^{2} \mathrm{By}$ construction of the functor $U(-)$, the above isomorphisms (6.3) occur already in the category NChow $(k)_{\mathbb{Z}}$ of noncommutative Chow motives with integral coefficients. Hence, using [5, Theorem 2.1] instead of [26, Theorems 1.3 and 1.7], we conclude that Proposition 6.4 holds also with $\mathbb{Q}$ replaced by $\mathbb{Z}[(4 m-4)$ !].
} 
Thanks to Proposition 6.4, $M(X)_{\mathbb{Q}}$ is of $\mathbb{Q}$-Lefschetz type. Consequently, since the identity of $M(X)_{\mathbb{Q}}$ decomposes into a sum of pairwise orthogonal idempotents of Lefschetz type, the rational cycle class map is an isomorphism

$$
c l: C H^{i}(X)_{\mathbb{Q}} \stackrel{\sim}{\longrightarrow} H^{2 i}(X(\mathbb{C}), \mathbb{Q}) .
$$

Now, recall from Reid's thesis [30, $\S 0.7$ and Corollary 3.15] that

$$
H^{2 i}(X(\mathbb{C}), \mathbb{Q}) \simeq \begin{cases}\mathbb{Q} & \text { if } i \neq m-1 \\ \mathbb{Q}^{\oplus(2 m+2)} & \text { if } i=m-1\end{cases}
$$

By combining (6.6)-(6.7), we then obtain the following isomorphisms

$$
C H_{i}(X)_{\mathbb{Q}} \simeq C H^{2 m-2-i}(X)_{\mathbb{Q}} \simeq\left\{\begin{array}{ll}
\mathbb{Q} & \text { if } i \neq m-1 \\
\mathbb{Q}^{\oplus(2 m+2)} & \text { if } i=m-1
\end{array} .\right.
$$

This clearly implies (6.2) and so the proof is finished.

Two even-dimensional quadrics. Let $n=2 m-1$ for some integer $m \geq 2$. Since we are intersecting two even-dimensional quadrics, we have $\operatorname{dim}(X)=2 m-3$ and $\kappa=m-2$. Hence, in order to prove Theorem 1.2, we need to show that

$$
C H_{i}(X)_{\mathbb{Q}} \simeq \mathbb{Q} \text { for } i<m-2 .
$$

Recall from Example 5.4 that the hyperelliptic curve naturally associated to $X$ is the discriminant double cover $C \rightarrow \mathbb{P}^{1}$ and that we have an equivalence $\operatorname{perf}\left(\mathbb{P}^{1}, \mathcal{C}_{0}\right) \simeq$ $\operatorname{perf}(C)$. The above semi-orthogonal decomposition (6.1) reduces then to

$$
\operatorname{perf}(X)=\left\langle\operatorname{perf}(C), \mathcal{O}_{X}(1), \ldots, \mathcal{O}_{X}(2 m-4)\right\rangle .
$$

From (6.10) we conclude automatically that $K_{0}(X)_{\mathbb{Q}} \simeq K_{0}(C)_{\mathbb{Q}} \oplus \mathbb{Q}^{2 m-4}$. Using the isomorphisms $K_{0}(X)_{\mathbb{Q}} \simeq C H^{*}(X)_{\mathbb{Q}}$ and $K_{0}(C)_{\mathbb{Q}} \simeq C H^{*}(C)_{\mathbb{Q}}$ we obtain

$$
C H^{*}(X)_{\mathbb{Q}} \simeq C H^{*}(C)_{\mathbb{Q}} \oplus \mathbb{Q}^{\oplus 2 m-4} .
$$

Now, recall from Voisin [35, Example 21.3.1] that the rational Chow ring of every curve $C$ admits the following decomposition $C H^{*}(C)_{\mathbb{Q}} \simeq \mathbb{Q} \oplus A_{0}(C)_{\mathbb{Q}} \oplus \mathbb{Q}$. By combining this decomposition with (6.11), one hence obtains

$$
C H^{*}(X)_{\mathbb{Q}} \simeq A_{0}(C)_{\mathbb{Q}} \oplus \mathbb{Q}^{\oplus 2 m-2} .
$$

Proposition 6.13. The inclusion $A_{0}(C)_{\mathbb{Q}} \subset C H^{*}(X)_{\mathbb{Q}}$ induces an isomorphism $A_{0}(C)_{\mathbb{Q}} \simeq A_{m-2}(X)_{\mathbb{Q}}$.

Proof. Thanks to the semi-orthogonal decomposition (6.10), the irreducible $k$ schemes $C$ and $X$ are related by the categorical data $(\star)$ of $\S 4$. Let us now verify all conditions of Theorem 4.3. The bilinear pairings (4.2) associated to $C$ and $X$ are non-degenerate since every curve and every complete intersection of smooth hypersurfaces in the projective space satisfy Grothendieck's standard conjecture of Lefschetz type; see André $[1, \S 5.2 .4 .5]$ and Grothendieck [14, §3]. The fact that the inclusion functor $\operatorname{perf}(C) \hookrightarrow \operatorname{perf}(X)$ is of Fourier-Mukai type was proved by Bondal-Orlov in [7, Theorem 2.7]. The remaining conditions of Theorem 4.3 are clearly verified. As a consequence, we obtain an isomorphism in $\mathrm{Ab}(k)_{\mathbb{Q}}$

$$
\tau: J(C) \stackrel{\sim}{\longrightarrow} \prod_{i=0}^{2 m-4} J_{a}^{i}(X)=J_{a}^{m-2}(X) \times \prod_{i \neq m-2} J_{a}^{i}(X) .
$$

This implies that $J_{a}^{\kappa}(X)=J_{a}^{m-2}(X) \subseteq \tau(J(C))$. As proved by Reid in [30, Theorem 4.14], we have $J_{a}^{m-2}(X) \simeq J(C)$ (as principally polarized abelian varieties). 
As a consequence, we conclude that $\tau(J(C)) \simeq J_{a}^{m-2}(X)$. Now, recall once again from Reid [30, Theorem 4.14] that the Abel-Jacobi maps

$$
A J^{1}: A^{1}(C)_{\mathbb{Z}} \rightarrow J^{0}(C)=J(C) \quad A J^{m-1}: A^{m-1}(X)_{\mathbb{Z}} \rightarrow J^{m-2}(X)
$$

give rise to the isomorphisms $A_{0}(C)_{\mathbb{Q}}=A^{1}(C)_{\mathbb{Q}} \simeq J(C)_{\mathbb{Q}}$ and $A_{m-2}(X)_{\mathbb{Q}}=$ $A^{m-1}(X)_{\mathbb{Q}} \simeq J_{a}^{m-2}(X)_{\mathbb{Q}}$. Making use of them and of $\tau$ we then obtain the desired isomorphism $A_{0}(C)_{\mathbb{Q}} \simeq A_{m-2}(X)_{\mathbb{Q}}$.

From Proposition 6.13 one obtains the following isomorphism

$$
C H^{*}(X)_{\mathbb{Q}} / A_{0}(C)_{\mathbb{Q}} \simeq \bigoplus_{i \neq m-2} C H_{i}(X)_{\mathbb{Q}} \oplus\left(C H_{m-2}(X)_{\mathbb{Q}} / A_{m-2}(X)_{\mathbb{Q}}\right) .
$$

On the other hand, (6.12) gives rise to the isomorphism

$$
C H^{*}(X)_{\mathbb{Q}} / A_{0}(C)_{\mathbb{Q}} \simeq \mathbb{Q}^{\oplus(2 m-2)} .
$$

Now, note that the intersection of the generic $m$-dimensional linear subspace of $\mathbb{P}^{2 m-1}$ with $X$ gives rise to a $(m-2)$-dimensional cycle $Z$ on $X$ with $\operatorname{deg}(Z) \neq 0$. This implies that $Z \notin A_{m-2}(X)_{\mathbb{Q}}$. Since $Z$ is non-torsion, we conclude also that $C_{m-2}(X)_{\mathbb{Q}} / A_{m-2}(X)_{\mathbb{Q}} \neq 0$. Similarly, by intersecting the generic $(i+2)$ dimensional linear subspace of $\mathbb{P}^{2 m-1}$ with $X$ one obtains a non-torsion $i$-dimensional cycle on $X$. This allows us to conclude that $C H_{i}(X)_{\mathbb{Q}} \neq 0$ for all $i \neq m-2$. Finally, a easy dimension counting argument (obtained by combining (6.15)-(6.16) with the fact that $\operatorname{dim}(X)+1=2 m-2)$ implies that $C H_{i}(X)_{\mathbb{Q}} \simeq \mathbb{Q}$ when $i \neq m-2$ and that $C H_{m-2}(X)_{\mathbb{Q}} / A_{m-2}(X)_{\mathbb{Q}} \simeq \mathbb{Q}$. This implies (6.9) and so the proof is finished.

\section{Proof of Theorem 1.2 - Case (ii)}

Let $n=2 m$ for some integer $m \geq 2$. Since we are intersecting three odddimensional quadrics, we have $\operatorname{dim}(X)=2 m-3$ and $\kappa=m-2$. Hence, in order to prove Theorem 1.2, we need to show that

$$
C H_{i}(X)_{\mathbb{Q}} \simeq \mathbb{Q} \text { for } i<m-2 .
$$

The linear span of these quadrics gives rise to a flat quadric fibration $q: Q \rightarrow \mathbb{P}^{2}$ of relative dimension $n-1$, As explained in $\S 5$, one obtains the semi-orthogonal decomposition

$$
\operatorname{perf}(X)=\left\langle\operatorname{perf}\left(\mathbb{P}^{2}, \mathcal{C}_{0}\right), \mathcal{O}_{X}(1), \ldots, \mathcal{O}_{X}(2 m-5)\right\rangle .
$$

From (7.2) we conclude automatically that

$$
K_{0}(X)_{\mathbb{Q}} \simeq K_{0}\left(\operatorname{perf}\left(\mathbb{P}^{2}, \mathcal{C}_{0}\right)\right)_{\mathbb{Q}} \oplus \mathbb{Q}^{\oplus 2 m-5} .
$$

Recall from Example 5.5 that $Q \rightarrow \mathbb{P}^{2}$ has a discriminant divisor, whose normalization $C$ comes with an étale double cover $\widetilde{C} \rightarrow C$; we write $\iota$ for the involution on $\widetilde{C}$. Thanks to the work of Bouali [8, Corollary 4.3], we have the following isomorphism

$$
C H^{*}(Q)_{\mathbb{Q}} \simeq C H^{*}\left(\mathbb{P}^{2}\right)_{\mathbb{Q}}^{\oplus 2 m} \oplus C H_{1}(\widetilde{C})_{\mathbb{Q}}^{-},
$$

where $C H_{1}(\widetilde{C})_{\mathbb{Q}}^{-}$stands for the $\iota$-anti-invariant part of $C H_{1}(\widetilde{C})_{\mathbb{Q}}$. As explained by Beauville in $[3, \S 2]$, the étale double cover $\widetilde{C} \rightarrow C$ gives rise to a natural splitting of $J(\widetilde{C})=C H_{1}(\widetilde{C})$ into the $\iota$-invariant part and the $\iota$-anti-invariant part, where the latter identifies with the Prym variety $\operatorname{Prym}(\widetilde{C} / C)$. In particular, we have an 
isomorphism $C H_{1}(\widetilde{C})_{\mathbb{Q}}^{-} \simeq A_{m}(Q)_{\mathbb{Q}}$. Consequently, since $A_{*}\left(\mathbb{P}^{2}\right)_{\mathbb{Q}}=0$, we conclude that $A_{*}(Q)_{\mathbb{Q}}=A_{m}(Q)_{\mathbb{Q}} \simeq C H_{1}(\widetilde{C})_{\mathbb{Q}}^{-}$.

Now, note that the general semi-orthogonal decomposition (5.1) reduces to

$$
\operatorname{perf}(Q)=\left\langle\operatorname{perf}\left(\mathbb{P}^{2}, \mathcal{C}_{0}\right), \operatorname{perf}\left(\mathbb{P}^{2}\right)_{1}, \ldots \operatorname{perf}\left(\mathbb{P}^{2}\right)_{2 m-1}\right\rangle .
$$

Moreover, thanks to Beilinson [4], perf $\left(\mathbb{P}^{2}\right)$ is generated by 3 exceptional objects.

Proposition 7.6. The Fourier-Mukai functor $\operatorname{perf}(Q) \rightarrow \operatorname{perf}\left(\mathbb{P}^{2}, \mathcal{C}_{0}\right) \hookrightarrow \operatorname{perf}(X)$ induces an isomorphism $A_{m}(Q)_{\mathbb{Q}} \simeq A_{m-2}(X)_{\mathbb{Q}}$.

Proof. Thanks to the above semi-orthogonal decompositions (7.2) and (7.5), one observes that the irreducible $k$-schemes $Q$ and $X$ are related by the categorical data $(\star)$ of $\S 4$. Let us now verify that all conditions of Theorem 4.3 are verified. The bilinear pairings (4.2) associated to $Q$ and $X$ are non-degenerate since Grothendieck's standard conjecture of Lefschetz type holds for every complete intersection of smooth hypersurfaces in the projective space (see Grothendieck [14, $\S 3])$ and for quadric fibrations over projective surfaces (see Vial [34, Theorem 7.4]). The functor $\operatorname{perf}(Q) \rightarrow \operatorname{perf}\left(\mathbb{P}^{2}, \mathcal{C}_{0}\right) \hookrightarrow \operatorname{perf}(X)$ is of Fourier-Mukai type since it is obtained by homological projective duality; see [21, Theorems 5.3 and 5.4]. The remaining conditions of Theorem 4.3(iii) are clearly verified. As a consequence, one obtains a well-defined isomorphism in $\mathrm{Ab}(k)_{\mathbb{Q}}$

$$
\tau: \prod_{i=0}^{2 m} J_{a}^{i}(Q) \stackrel{\sim}{\longrightarrow} \prod_{i=0}^{2 m-4} J_{a}^{i}(X) .
$$

Thanks to Bouali's computation (7.4), we have $J_{a}^{i}(Q)=0$ when $i \neq m$. Consequently, the above isomorphism (7.7) reduces to

$$
\tau: J_{a}^{m}(Q) \stackrel{\sim}{\longrightarrow} \prod_{i=0}^{2 m-4} J_{a}^{i}(X) .
$$

Beauville proved in [3, Theorems 2.1 and 6.3] that $J_{a}^{\kappa}(X)=J_{a}^{m-2}(X)$ is isomorphic, as a principally polarized abelian variety, to the $\operatorname{Prym}$ variety $\operatorname{Prym}(\widetilde{C} / C) \simeq$ $J_{a}^{m}(Q)$. We hence conclude that $\tau\left(J_{a}^{m}(Q)\right)=J_{a}^{m-2}(X)$ and that $J_{a}^{i}(X)$ is isogenous to zero when $i \neq m-2$. Now, recall once again from Beauville [3, Proposition 3.3 and Theorem 6.3] that the Abel-Jacobi maps

$$
A J^{m}: A^{m+1}(Q)_{\mathbb{Z}} \rightarrow J^{m}(Q) \quad A J^{m-2}: A^{m-1}(X)_{\mathbb{Z}} \rightarrow J^{m-2}(X)
$$

give rise to the isomorphisms $A_{m}(Q)_{\mathbb{Q}}=A^{m+1}(Q)_{\mathbb{Q}} \simeq J^{m+1}\left(Q^{\prime}\right)_{\mathbb{Q}}$ and $A_{m-2}(X)_{\mathbb{Q}}=$ $A^{m-1}(X)_{\mathbb{Q}} \simeq J^{m-2}(X)_{\mathbb{Q}}$. Making use of them and of $\tau$ one then obtains the desired isomorphism $A_{m}(Q)_{\mathbb{Q}} \simeq A_{m-2}(X)_{\mathbb{Q}}$.

Lemma 7.9. There is an isomorphism of $\mathbb{Q}$-vector spaces

$$
K_{0}\left(\operatorname{perf}\left(\mathbb{P}^{2}, \mathcal{C}_{0}\right)\right)_{\mathbb{Q}} \simeq A_{m}(Q)_{\mathbb{Q}} \oplus \mathbb{Q}^{\oplus 3} .
$$

Proof. Using the semi-orthogonal decomposition (7.5), we obtain the isomorphism

$$
K_{0}(Q)_{\mathbb{Q}} \simeq K_{0}\left(\operatorname{perf}\left(\mathbb{P}^{2}, \mathcal{C}_{0}\right)\right)_{\mathbb{Q}} \oplus \mathbb{Q}^{\oplus 6 m-3} .
$$

Proposition 7.6 implies that $A_{m}(Q)_{\mathbb{Q}} \subset K_{0}\left(\operatorname{perf}\left(\mathbb{P}^{2}, \mathcal{C}_{0}\right)\right)_{\mathbb{Q}}$ via the canonical isomorphism $K_{0}(Q)_{\mathbb{Q}} \simeq C H^{*}(Q)_{\mathbb{Q}}$. Hence, using the isomorphism $C H^{*}\left(\mathbb{P}^{2}\right)_{\mathbb{Q}} \simeq \mathbb{Q}^{3}$, we conclude from Bouali's computation (7.4) that

$$
C H^{*}(Q)_{\mathbb{Q}} \simeq A_{m}(Q)_{\mathbb{Q}} \oplus \mathbb{Q}^{6 m} .
$$


The desired isomorphism (7.10) is then obtained by comparing (7.11) with (7.12) via the canonical isomorphism $K_{0}(Q)_{\mathbb{Q}} \simeq C H^{*}(Q)_{\mathbb{Q}}$.

By combining the natural isomorphism $K_{0}(X)_{\mathbb{Q}} \simeq C H^{*}(X)_{\mathbb{Q}}$ with $(7.3)$ and (7.10), one concludes that

$$
C H^{*}(X)_{\mathbb{Q}} \simeq A_{m}(Q)_{\mathbb{Q}} \oplus \mathbb{Q}^{\oplus 2 m-2} .
$$

From Proposition 7.6 one obtains the following isomorphism

$$
C H^{*}(X)_{\mathbb{Q}} / A_{m}(Q)_{\mathbb{Q}} \simeq \bigoplus_{i \neq m-2} C H_{i}(X)_{\mathbb{Q}} \oplus\left(C H_{m-2}(X)_{\mathbb{Q}} / A_{m-2}(X)_{\mathbb{Q}}\right)
$$

On the other hand, (7.13) gives rise to the isomorphism

$$
C H^{*}(X)_{\mathbb{Q}} / A_{m}(Q)_{\mathbb{Q}} \simeq \mathbb{Q}^{\oplus 2 m-2} .
$$

Now, note that the intersection of the generic $(m+1)$-dimensional linear subspace of $\mathbb{P}^{2 m-1}$ with $X$ gives rise to a $(m-2)$-dimensional cycle $Z$ on $X$ with $\operatorname{deg}(Z) \neq 0$. Hence, $Z \notin A_{m-2}(X)_{\mathbb{Q}}$. Since $Z$ is non-torsion, we conclude that $C H_{m-2}(X)_{\mathbb{Q}} / A_{m-2}(X)_{\mathbb{Q}} \neq 0$. Similarly, by intersecting the generic $(i+3)$-dimensional linear subspace of $\mathbb{P}^{2 m-1}$ with $X$ one obtains a non-torsion $i$-dimensional cycle on $X$. This allows us to conclude that $C H_{i}(X)_{\mathbb{Q}} \neq 0$ for all $i \neq m-2$. Finally, a easy dimension counting argument (obtained by combining (7.14)-(7.15) with the fact that $\operatorname{dim}(X)+1=2 m-2)$ implies that $C H_{i}(X)_{\mathbb{Q}} \simeq \mathbb{Q}$ when $i \neq m-2$ and that $C H_{m-2}(X)_{\mathbb{Q}} / A_{m-2}(X)_{\mathbb{Q}} \simeq \mathbb{Q}$. This implies $(7.1)$ and so the proof is finished.

\section{Proof of Theorem 1.4}

Two odd-dimensional quadrics. Let $n=2 m$ for some integer $m \geq 2$. Since we are intersecting two odd-dimensional quadrics, we have $\operatorname{dim}(X)=2 m-2$. Recall from the proof of Proposition 7.6 that $X$ satisfies Grothendieck's standard conjecture of Lefschetz type, and from (6.3) that we have the motivic decomposition $N C(X)_{\mathbb{Q}} \simeq \mathbf{1}_{\mathbb{Q}}^{\oplus 4 m}$. By applying to it the (additive) Jacobian functor $\mathbf{J}(-)$, we obtain the following isomorphisms in $\mathrm{Ab}(k)_{\mathbb{Q}}$

$$
\prod_{i=0}^{2 m-3} J_{a}^{i}(X) \simeq \mathbf{J}\left(N C(X)_{\mathbb{Q}}\right) \simeq \bigoplus_{i=1}^{4 m} \mathbf{J}\left(\mathbf{1}_{\mathbb{Q}}\right) \simeq \bigoplus_{i=1}^{4 m} \mathbf{J}\left(N C(\operatorname{Spec}(k))_{\mathbb{Q}}\right) \simeq 0 .
$$

This clearly implies that $J_{a}^{i}(X)=0$ for every $i$.

Two even-dimensional quadrics. Let $n=2 m-1$ for some integer $m \geq 2$. Since we are intersecting two even-dimensional quadrics, we have $\operatorname{dim}(X)=2 m-3$ and $\kappa=m-2$. Recall from (6.10) the construction of the semi-orthogonal decomposition

$$
\operatorname{perf}(X)=\left\langle\operatorname{perf}(C), \mathcal{O}_{X}(1), \ldots, \mathcal{O}_{X}(2 m-4)\right\rangle
$$

and from the proof of Proposition 7.6 that $C$ and $X$ satisfy Grothendieck's standard conjecture of Lefschetz type. Thanks to Proposition 3.1, (8.1) gives rise to the motivic decomposition $N C(X)_{\mathbb{Q}} \simeq N C(C)_{\mathbb{Q}} \oplus \mathbf{1}_{\mathbb{Q}}^{\oplus 2 m-4}$. By applying to it the (additive) Jacobian functor $\mathbf{J}(-)$, we obtain the following isomorphisms in $\mathrm{Ab}(k)_{\mathbb{Q}}$

$$
\prod_{i=0}^{2 m-4} J_{a}^{i}(X) \simeq \mathbf{J}\left(N C(X)_{\mathbb{Q}}\right) \simeq \mathbf{J}\left(N C(C)_{\mathbb{Q}}\right) \oplus \mathbf{J}\left(N C(\operatorname{Spec}(k))_{\mathbb{Q}}\right)^{\oplus 2 m-4} \simeq J(C) .
$$


Now, recall from the proof of Proposition 6.13 that

$$
J_{a}^{m-2}(X)_{\mathbb{Q}} \simeq A_{m-2}(X)_{\mathbb{Q}} \simeq A_{0}(C)_{\mathbb{Q}} \simeq J(C)_{\mathbb{Q}} .
$$

By combining the above two isomorphisms we conclude that $J_{a}^{i}(X)$ is isogenous to zero (and hence is zero) for $i \neq \kappa$.

Three odd-dimensional quadrics. Let $n=2 m$ for some integer $m \geq 2$. Since we are intersecting three odd-dimensional quadrics, we have $\operatorname{dim}(X)=2 m-3$ and $\kappa=m-2$. We start by recalling a consequence of Conjecture 1.1, which is based on Bloch-Srinivas methods of decomposition of the diagonal.

Proposition 8.2. (Paranjape [28, Theorem 6.3]) Let $Y$ be a d-dimensional complete intersection satisfying Conjecture 1.1. In this case we have:

(i) $C H_{i}(Y)_{\mathbb{Q}} \simeq \mathbb{Q}$ for every $i<\kappa$;

(ii) $C H_{i}(Y)_{\mathbb{Q}} \simeq \mathbb{Q}$ for every $i>d-\kappa$;

(iii) $C H_{d-\kappa}(Y)_{\mathbb{Q}}$ is a finite dimensional $\mathbb{Q}$-vector space.

Corollary 8.3. Let $X$ be a complete intersection of three odd-dimensional quadrics. In this case, $C H_{i}(X)_{\mathbb{Q}}$ is a finite dimensional $\mathbb{Q}$-vector space for every $i \neq m-2$.

Proof. Recall from above that $\kappa=m-2$ and $d=2 m-3$. Hence, the proof follows from the combination of Theorem 1.2 with Proposition 8.2.

We now have all the ingredients necessary for the proof. In codimensional notation Corollary 8.3 shows that $C H^{i}(X)_{\mathbb{Q}}$ is a finite dimensional $\mathbb{Q}$-vector space for every $i \neq m-1$. This implies that the subspaces $A^{i}(X)_{\mathbb{Q}} \subset C H^{i}(X)_{\mathbb{Q}}, i \neq m-1$, are finite dimensional that that their images under the Abel-Jacobi maps

$$
A J^{i-1}: A^{i}(X)_{\mathbb{Q}} \rightarrow J^{i-1}(X)_{\mathbb{Q}} \quad i \neq m-1
$$

are isogeneous to zero. We conclude then that $J_{a}^{i}(X)=0$ for every $i \neq m-2$.

Remark 8.4. The above proof can be easily adapted to the case of a complete intersection of two quadrics.

\section{Proof of Corollary 2.1}

Two odd-dimensional quadrics. Let $n=2 m$ for some integer $m \geq 2$. Since we are intersecting two odd-dimensional quadrics, we have $d:=\operatorname{dim}(X)=2 m-$ 2. As explained in the proof of Proposition 6.4, there exists a choice of integers $l_{1}, \ldots, l_{4 m} \in\{0, \ldots, d\}$ giving rise to an isomorphism

$$
M(X)_{\mathbb{Q}} \simeq \mathbf{L}^{\otimes l_{1}} \oplus \cdots \oplus \mathbf{L}^{\otimes l_{4 m}} .
$$

Note that this isomorphism implies automatically that $M(X)_{\mathbb{Q}}$ is Kimura-finite. Using the following computation (see (6.8))

$$
C H_{i}(X)_{\mathbb{Q}} \simeq \begin{cases}\mathbb{Q} & \text { if } \quad i \neq m-1 \\ \mathbb{Q}^{\oplus(2 m+2)} & \text { if } \quad i=m-1,\end{cases}
$$

one concludes that

$$
M^{i}(X)_{\mathbb{Q}}= \begin{cases}\mathbf{L}^{\otimes \frac{i}{2}} & \text { if } 0 \leq i \leq 4 m-4, i \neq 2 m-2, i \text { even } \\ \left(\mathbf{L}^{\otimes m-1}\right)^{\oplus 2 m+2} & \text { if } i=2 m-2 \\ 0 & \text { otherwise. }\end{cases}
$$

Finally, using the fact that $d=2 m-2$, we observe that (9.1) agrees with (2.2). 
Two even-dimensional or three odd-dimensional quadrics. Let $m$ be an integer $\geq 2$. Since we are intersecting two even-dimensional or three odd-dimensional quadrics, we have $d:=\operatorname{dim}(X)=2 m-3$ and $\kappa=m-2$. Recall that the group $A_{i}(Y)_{\mathbb{Q}}$ of a $k$-scheme $Y$ is called rationally representable if there exists a curve $\Gamma$ and an algebraic surjective map $A_{0}(\Gamma)_{\mathbb{Q}} \rightarrow A_{i}(Y)_{\mathbb{Q}}$.

Theorem 9.2. (Vial [33, Theorem 4]) Given an irreducible k-scheme $Y$ of dimension $d$, the following statements are equivalent:

(i) One has the following motivic decomposition

$$
M^{j}(Y)_{\mathbb{Q}}= \begin{cases}\left(\mathbf{L}^{\otimes l}\right)^{\oplus a_{l}} & \text { if } j=2 l \\ M^{1}\left(J_{a}^{l}(Y)\right)_{\mathbb{Q}}(l-d) & \text { if } j=2 l+1\end{cases}
$$

for some integer $a_{l} \geq 1$.

(ii) The groups $A_{i}\left(Y_{\mathbb{C}}\right)_{\mathbb{Q}}, 0 \leq i \leq d$, are rationally representable.

Now, recall from Theorem 1.4(ii) that $J_{a}^{i}(X)=0$ for $i \neq m-1$. This implies that the groups $A_{i}(X)_{\mathbb{Q}}$ are trivial for $i \neq m-2$ and consequently that they are rationally representable. In the case of a complete intersection of two evendimensional quadrics, we have an algebraic isomorphism $A_{m-2}(X)_{\mathbb{Q}} \simeq A_{0}(C)_{\mathbb{Q}} \simeq$ $J(C)_{\mathbb{Q}}$; consult Reid [30, Theorem 4.14] for details. In the case of a complete intersection of three odd-dimensional quadrics, we have an algebraic isomorphism $A_{m-2}(X)_{\mathbb{Q}} \simeq A_{1}\left(Q^{\prime}\right)_{\mathbb{Q}} \simeq \operatorname{Prym}(\widetilde{C} / C)_{\mathbb{Q}}$ which is induced by an algebraic surjective map $A_{0}(\widetilde{C}) \rightarrow A_{m-2}(X)$; consult Beauville [3, Theorems 3.1 and 6.3] for details. Therefore, in both cases, the group $A_{m-2}(X)_{\mathbb{Q}}$ (and hence $\left.A_{m-2}\left(X_{\mathbb{C}}\right)_{\mathbb{Q}}\right)$ is also rationally representable. This implies condition (ii) of Theorem 9.2. As explained in the proof of Theorem 1.2, we have $C H_{i}(X)_{\mathbb{Q}} \simeq \mathbb{Q}$ for $i \neq m-2$ and $C H_{m-2}(X)_{\mathbb{Q}} / A_{m-2}(X)_{\mathbb{Q}} \simeq \mathbb{Q}$. As a consequence, all the $a_{i}$ 's are equal to 1 and the above decomposition (9.3) agrees with (2.3).

\section{Proof of Corollary 2.4}

Let $X$ be a $k$-scheme. Recall that the Chow group $C H_{i}(X)_{\mathbb{Q}}$ has niveau $\leq r$ if there exists a closed subscheme $Z \subset X$ of dimension $i+r$ such that the proper pushforward map $C H_{i}\left(Z_{\mathbb{C}}\right) \rightarrow C H_{i}\left(X_{\mathbb{C}}\right)$ is surjective.

Theorem 10.1. (Vial [34, Theorem 7.1]) Let $X$ be an irreducible $k$-scheme of dimension d. Assume that the Chow groups $\mathrm{CH}_{0}\left(X_{\mathbb{C}}\right), \ldots, \mathrm{CH}_{l}\left(X_{\mathbb{C}}\right)$ have niveau $\leq n$. Under these assumptions, the following holds:

(i) If $n=3$ and $l=\left\lfloor\frac{d-4}{2}\right\rfloor$, then $X$ satisfies Hodge's conjecture;

(ii) If $n=2$ and $l=\left\lfloor\frac{d-3}{2}\right\rfloor$, then $X$ satisfies Lefschetz's standard conjecture;

(iii) If $n=1$ and $l=\left\lfloor\frac{d-3}{2}\right\rfloor$, then $X$ satisfies Murre's conjecture;

(iv) If $n=1$ and $l=\left\lfloor\frac{d-2}{2}\right\rfloor$, then $M(X)_{\mathbb{Q}}$ is Kimura-finite, where $\lfloor-\rfloor$ stands for the floor function.

Theorem 10.2. (Vial [34, Theorem 6.10]) Let $\pi: X \rightarrow B$ be a smooth dominant flat morphism between $k$-schemes. Assume that $C H_{i}\left(X_{b}\right)=\mathbb{Q}$ for all $i<l$ and all closed point $b \in B$. Then, $C H_{i}(X)_{\mathbb{Q}}$ has niveau $\leq \operatorname{dim}(B)$ for all $i<l$.

We now have all the ingredients needed for the proof of Corollary 2.4. The proof will consist on verifying the conditions of Theorems 10.1-10.2. Recall that $f: Y \rightarrow$ $B$ is a smooth dominant flat morphism whose fibers are complete intersections either 
of two quadrics or of three odd-dimensional quadrics. In these cases, Theorem 1.2 implies that $C H_{i}\left(Y_{b}\right)_{\mathbb{Q}} \simeq \mathbb{Q}$ for $i<\kappa=\left[\operatorname{dim}\left(Y_{b}\right) / 2\right]$. As a consequence, $C H_{l}(Y)$ has niveau $\leq \operatorname{dim}(B)$ for $l \leq\left[\frac{d-\operatorname{dim}(B)-1}{2}\right]$. By applying Theorem 10.1 we then obtain the items (i)-(iii) of Corollary 2.4.

\section{REFERENCES}

[1] Y. André, Une introduction aux motifs (motifs purs, motifs mixtes, périodes). Panoramas et Synthèses 17. Société Mathématique de France, Paris, 2004.

[2] A. Auel, M. Bernardara and M. Bolognesi, Fibrations in complete intersections of quadrics, Clifford algebras, derived categories, and rationality problems. Journal de mathématiques pures et appliquées 102 (2014), 249-291.

[3] A. Beauville, Variétés de Prym et jacobiennes intermédiaires. Ann. Sci. de l'ENS 10 (1977), 309-391.

[4] A. Beilinson, Coherent sheaves on $\mathbb{P}^{n}$ and problems in linear algebra. (Russian) Funktsional. Anal. i Prilozhen. 12 (1978), no. 3, 68-69.

[5] M. Bernardara and G. Tabuada, Relations between the Chow motive and the noncommutative motive of a smooth projective variety. Available at arXiv:1303.3172v(3). To appear in Journal of Pure and Applied Algebra.

[6] _ From semi-orthogonal decompositions to polarized intermediate Jacobians via Jacobians of noncommutative motives. Available at arXiv:1305.4687.

[7] A. Bondal and D. Orlov, Semi-orthogonal decomposition for algebraic varieties. Available at arXiv math.AG/9506012.

[8] J. Bouali, Motives of quadric bundles. Available at arXiv:1310.2782 (2013).

[9] F. Campana, T. Peternell and A.V. Pukhlikov, The generalized Tsen theorem and rationally connected Fano fibrations. Mat. Sb. 193 (2002), no. 10, 49-74.

[10] R. Elman, N. Karpenko and A. Merkurjev, The algebraic and geometric theory of quadratic forms, American Mathematical Society Colloquium Publications, vol. 56, American Mathematical Society, Providence, RI, 2008.

[11] H. Esnault, M. Levine and E. Viehweg, Chow groups of projective varieties of very small degree. Duke Math. J. 87 (1997), no. 1, 29-58.

[12] T. Graber, J. Harris and J. Starr, Families of rationally connected varieties. J. Amer. Math. Soc. 16 (2003), no. 1, 57-67.

[13] P. Griffiths, On the periods of certain rational integrals I, II. Ann. of Math. (2) 90 (1969), 460-495.

[14] A. Grothendieck, Standard conjectures on algebraic cycles. 1969 Algebraic Geometry (Internat. Colloq., Tata Inst. Fund. Res., Bombay, 1968) pp. 193-199. Oxford Univ. Press, London.

[15] J. Harris, Algebraic geometry, Graduate Texts in Mathematics, vol. 133, Springer-Verlag, New York, 1995, A first course, Corrected reprint of the 1992 original.

[16] B. Keller, On differential graded categories. International Congress of Mathematicians (Madrid), Vol. II, 151-190, Eur. Math. Soc., Zürich, 2006.

[17] M. Kontsevich, Noncommutative motives. Talk at the Institute for Advanced Study on the occasion of the $61^{\text {st }}$ birthday of Pierre Deligne, October 2005 . Video available at http://video.ias.edu/Geometry-and-Arithmetic.

[18] _ Mixed noncommutative motives. Talk at the Workshop on Homological Mirror Symmetry, Miami, 2010. Notes available at www-math.mit.edu/auroux/frg/miami10-notes.

[19] - Notes on motives in finite characteristic. Algebra, arithmetic, and geometry: in honor of Yu. I. Manin. Vol. II, 213-247, Progr. Math., 270, Birkhuser Boston, MA, 2009.

[20] A. Kuznetsov, Homological projective duality. Publ. IHÉS 105 (2007), 157-220.

[21] Derived categories of quadric fibrations and intersections of quadrics. Advances in Math. 218 (2008), no. 5, 1340-1369.

[22] D. Leep The Amer-Brumer Theorem over arbitrary fields, preprint, 2007.

[23] V. Lunts and D. Orlov, Uniqueness of enhancement for triangulated categories. J. Amer. Math. Soc. 23 (2010), no. 3, 853-908.

[24] Ju I. Manin, Correspondences, motifs and monoidal transformations Mat. Sb. (N.S.) 77 (119) (1968), 475-507. 
[25] M. Marcolli and G. Tabuada, Jacobians of noncommutative motives. Moscow Mathematical Journal 14 (2014), no. 3, 577-594.

[26] - From exceptional collection to motivic decompositions via noncommutative motives. J. Reine Angew. Math. 701 (2015), 153-167.

[27] A. Otwinowska, Remarques sur les cycles de petite dimension de certaines intersections complètes, C. R. Acad. Sci. Paris, 329 (1999), Série I, p. 141-146.

[28] K. Paranjape, Cohomological and cycle-theoretic connectivity. Ann. of Math. (2) 139 (1994), no. 3, 641-660.

[29] A. Polishchuk, Holomorphic bundles on 2-dimensional noncommutative toric orbifolds. Noncommutative geometry and number theory, 341-359, Aspects Math., E37, Vieweg, Wiesbaden, 2006.

[30] M. Reid, The complete intersection of two or more quadrics. Ph.D. Thesis, Cambridge, 1972. Available at http://homepages.warwick.ac.uk/ masda/3folds/qu.pdf

[31] G. Tabuada, A guided tour through the garden of noncommutative motives. Clay Mathematics Proceedings, Volume 16 (2012), 259-276.

[32] — Additive invariants of dg categories. Int. Math. Res. Not. 53 (2005), 3309-3339.

[33] C. Vial, Projectors on the intermediate algebraic Jacobians. New York J. Math. 19 (2013), 793-822.

[34] _ Algebraic cycles and fibrations. Documenta Math. 18 (2013), 1521-1553.

[35] C. Voisin, Théorie de Hodge et géométrie algébrique complexe. Cours Spécialisés 10, Société Mathématique de France, Paris, 2002.

Institut de Mathématiques de Toulouse ; UMR5219 - Université de Toulouse ; CNRS - UPS IMT, F-31062 Toulouse Cedex 9, France

E-mail address: marcello.bernardara@math.univ-toulouse.fr

URL: http://www.math.univ-toulouse.fr/〜mbernard/

Gonçalo Tabuada, Department of Mathematics, Mit, Cambridge, Ma 02139, USA

E-mail address: tabuada@math.mit.edu

URL: http://math.mit.edu/ ${ }^{\sim}$ tabuada/ 